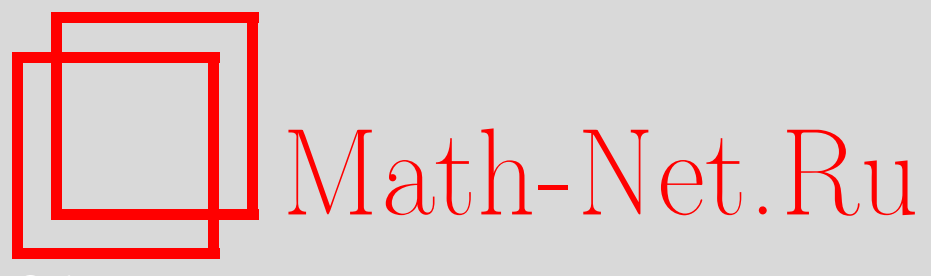

Х. Д. Икрамов, О комплексных матрицах, унитарно подобных вещественным матрицам, Матем. заметки, 2010, том 87, выпуск 6, 840-847

DOI: https://doi.org/10.4213/mzm8532

Использование Общероссийского математического портала Math-Net.Ru подразумевает, что вы прочитали и согласны с пользовательским соглашением http://www . mathnet.ru/rus/agreement

Параметры загрузки:

IP : 35.174 .16 .151

26 апреля 2023 г., 16:20:00

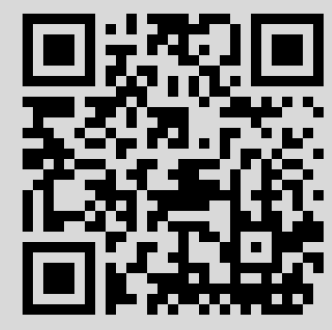


Том 87 выпуск 6 июнь 2010

УДК 512.643

\section{О комплексных матрицах, унитарно подобных вещественным матрицам}

\section{Х. Д. Икрамов}

Известны условия, при которых комплексная $(n \times n)$-матрица $A$ может быть посредством подобия преобразована в вещественную матрицу. Возможно ли такое преобразование осуществить посредством не произвольного, а унитарного подобия? На этот вопрос в данной статье дан такой ответ: матрица $A \in M_{n}(\mathbb{C})$ тогда и только тогда может быть овеществлена унитарным подобием, когда $A$ и $\bar{A}$ унитарно подобны и подобие между ними можно осуществить посредством матрицы $P$, одновременно унитарной и симметричной. Обсуждены способы эффективной проверки этого критерия.

Библиография: 5 названий.

1. Введение. Пусть $M_{n}(\mathbb{C})$ - пространство комплексных $(n \times n)$-матриц. Предположим, что для матрицы $A \in M_{n}(\mathbb{C})$ существует подобная ей вещественная матрица

$$
B=Q^{-1} A Q
$$

Тогда $A$ должна иметь те же особенности спектра и жордановой структуры, какими обладают вещественные матрицы. Перечислим эти особенности:

а) если невещественное число $\lambda$ является собственным значением для $A$, то $\bar{\lambda}-$ также собственное значение, причем той же кратности;

б) число и порядки жордановых клеток, относящихся к $\bar{\lambda}$, те же, что и для $\lambda$. Условия а) и б) необходимы для подобия (1). Но эти же условия достаточны для того, чтобы $A$ можно было подобием перевести в вещественную матрицу. В самом деле, выполним подобие, преобразующее $A$ в ее жорданову форму $J$. Если $A$ имеет чисто вещественный спектр, то $J$ - вещественная матрица и доказывать нечего. Предположим, что имеются и невещественные собственные значения. Выбирая подходящий порядок жордановых клеток, можем считать, что в матрице $J$ вслед за каждой клеткой $J_{k}(\lambda)$, где $\lambda=\sigma+i \tau, \tau \neq 0$, стоит клетка $J_{k}(\bar{\lambda})$. Здесь $J_{k}(\lambda)-$ стандартное обозначение жордановой клетки порядка $k$, относящейся к числу $\lambda$.

Матрица

$$
J_{k}(\lambda) \oplus J_{k}(\bar{\lambda})
$$


подобна вещественной блочной матрице

$$
\left(\begin{array}{ccccc}
C & I_{2} & & & \\
& C & I_{2} & & \\
& & \cdots & \cdots & \\
& & & \cdots & \\
& & & & C
\end{array}\right)
$$

порядка $2 k$ с диагональным блоком

$$
C=\left(\begin{array}{cc}
\sigma & \tau \\
-\tau & \sigma
\end{array}\right)
$$

(см. об этом подробнее $[1 ; \S 6.6])$. Символ $I_{n}$ есть стандартное обозначение единичной матрицы порядка $n$. Заменяя в $J$ каждую пару клеток вида (2) соответствующей матрицей (3), получим вещественную матрицу, подобную $A$ (и называемую ее вещественной жордановой формой).

Пусть матрица $A \in M_{n}(\mathbb{C})$ обладает свойствами а) и б). Зададимся вопросом о том, можно ли для соотношения (1), где $B$ - вещественная матрица, подобрать унитарную матрицу $Q$. Иначе говоря, можно ли овеществить $A$ посредством унитарного подобия? Множество $(n \times n)$-матриц $A$, для которых ответ на этот вопрос положителен, будем обозначать через $\mathscr{\mathscr { R }}{ }_{n}$.

В п. 3 мы устанавливаем следующий критерий для включения $A \in \mathscr{U} \mathscr{R}_{n}$.

Теорема 1. Матрица $A \in M_{n}(\mathbb{C})$ тогда и толъко тогда может бытъ овеществлена унитарным подобием, когда $A$ и $\bar{A}$ унитарно подобны и подобие между ними можно осуществить посредством матрицы $P$, одновременно унитарной $и$ симметричной.

Как обычно, черта над символом матрицы обозначает поэлементное комплексное сопряжение.

Наличие или отсутствие унитарного подобия между матрицами $A, B \in M_{n}(\mathbb{C})$ можно проверить с помощью классической теоремы Шпехта (см. [2; теорема 2.2.6]).

Теорема 2 (Шпехт). Матрицы $A, B \in M_{n}(\mathbb{C})$ унитарно подобны тогда и толъко тогда, когда

$$
\operatorname{tr} W\left(A, A^{*}\right)=\operatorname{tr} W\left(B, B^{*}\right)
$$

для любого слова $W(s, t)$ от двух некоммутирующих переменных $s$ u $t$.

Согласно уточнению этого критерия, найденному Пирси [3], равенства (5) достаточно проверить для слов степени не больше $2 n^{2}$. При этом степенью слова

$$
W(s, t)=s^{m_{1}} t^{n_{1}} s^{m_{2}} t^{n_{2}} \cdots s^{m_{k}} t^{n_{k}}
$$

называется число

$$
m_{1}+n_{1}+\cdots+m_{k}+n_{k} .
$$

Проверка второго условия теоремы 1 (т.е. симметрии трансформирующей унитарной матрицы $P$ ) является значительно более сложным вопросом. Дело в том, что критерий Шпехта может установить существование матрицы $P$, но он не дает метода ее построения. 
В настоящее время автору не известен способ эффективной проверки этого условия. В связи с этим в п. 4 рассмотрен специальный случай, когда критерий теоремы 1 выполнен.

Назовем матрицу $A \in M_{n}(\mathbb{C})$ неприводимой, если $A$ и $A^{*}$ не имеют нетривиальных общих инвариантных подпространств. Иначе говоря, $A$ неприводима, если не существует унитарного подобия, которое превращало бы $A$ в прямую сумму матриц меньшего порядка. Эффективный способ проверки неприводимости указан в [4].

В п. 4 доказано следующее утверждение.

ТеОрема 3. Если неприводимая матрица $A \in M_{n}(\mathbb{C})$ нечетного порядка п унитарно подобна матрище $\bar{A}$, то $A \in \mathscr{U} \mathscr{R}_{n}$.

В п. 2, содержащем вспомогательные сведения, и в обсуждении, сопровождающем теорему 3, мы объясняем, почему условие нечетности порядка существенно.

2. Предварительные сведения. Матрица $A \in M_{n}(\mathbb{C})$ называется псевдоинволющией (coninvolution), если

$$
A \bar{A}=I_{n} .
$$

Нам потребуется следующий факт, связанный с псевдоинволюциями (см. [2; лемма 4.6.9]).

Теорема 4. Матрица $A \in M_{n}(\mathbb{C})$ тогда и только тогда является псевдоинволючией, когда А может быть представлена в виде

$$
A=S \bar{S}^{-1}
$$

для некоторой невырожденной матрищъ $S \in M_{n}(\mathbb{C})$.

В доказательстве теоремы 1 используется классический, но, тем не менее, не слишком широко известный факт, называемый факторизацией Такаги (см. [2; следствие 4.4.4]).

Теорема 5. Для всякой симметричной матрицы $A \in M_{n}(\mathbb{C})$ найдутся унитарная матрица $U \in M_{n}(\mathbb{C})$ и диагональная матрица

$$
\Sigma=\operatorname{diag}\left(\sigma_{1}, \ldots, \sigma_{n}\right)
$$

такие, чmо

$$
A=U \Sigma U^{T}
$$

Матрицу $U$ в теореме 5 можно выбрать так, чтобы $\Sigma$ была неотрицательной матрицей. В этом случае диагональными элементами $\sigma_{1}, \ldots, \sigma_{n}$ будут сингулярные числа матрицы $A$ (т.е. неотрицательные квадратные корни из собственных значений матрицы $\left.A A^{*}=A \bar{A}\right)$.

Пусть $n$ - четное число: $n=2 m$. Положим

$$
J=\left(\begin{array}{cc}
0 & I_{m} \\
-I_{m} & 0
\end{array}\right) .
$$

Назовем блочным кватернионом всякую матрицу $A \in M_{n}(\mathbb{C})$, удовлетворяющую соотношению

$$
\bar{A} J=J A \text {. }
$$


Если блочный кватернион $A$ представить в виде

$$
A=\left(\begin{array}{ll}
A_{11} & A_{12} \\
A_{21} & A_{22}
\end{array}\right)
$$

с блоками размера $m \times m$, то эти блоки связаны соотношениями

$$
A_{21}=-\bar{A}_{12}, \quad A_{22}=\bar{A}_{11} .
$$

Из определения (11) видно, что блочный кватернион $A$ унитарно подобен матрице $\bar{A}$. Однако трансформирующая матрица $J$ кососимметрична, а не симметрична, как того требует теорема 1 . Поэтому ни откуда не следует, что $A \in \mathscr{U} \mathscr{R}_{n}$. Если все же $A \in \mathscr{U} \mathscr{R}_{n}$, то $A$ обязательно является приводимой матрицей. В самом деле, пусть

$$
A=Q R Q^{*}
$$

где

$$
Q Q^{*}=I_{n} \quad \text { и } \quad R \in M_{n}(\mathbb{R}) .
$$

Подставляя (14) в (11), имеем

$$
\bar{Q} R Q^{T} J=J Q R Q^{*}
$$

или

$$
R\left(Q^{T} J Q\right)=\left(Q^{T} J Q\right) R
$$

Как и $J$, матрица

$$
K=Q^{T} J Q
$$

унитарная и кососимметричная. Поэтому равенство

$$
K=\alpha I_{n}
$$

невозможно ни при каком $\alpha$. Следовательно, $K$ имеет нетривиальную пару ортогональных инвариантных подпространств, отвечающих взаимно дополнительным частям ее спектра. В силу (15) те же подпространства инвариантны относительно $R$, т.е. являются общими инвариантными подпространствами для $R$ и $R^{*}$. Итак, $R$ приводима, а тогда приводима и унитарно подобная ей матрица $A$.

Пусть $P$ - произвольная матрица, одновременно унитарная и кососимметричная. (Это сочетание свойств возможно только для четного $n$; оно имеет место, в частности, для матриц $J$ и $K$.) Из соотношений

$$
P P^{*}=I_{n} \quad \text { и } \quad P^{T}=-P
$$

следует, что

$$
P \bar{P}=-I_{n}
$$

Всякая матрица $P$, удовлетворяющая условию (17), называется косой псевдоинволющией (skew-coninvolution).

По аналогии с (11) определим для унитарной кососимметричной матрицы $P$ класс матриц $B$ таких, что

$$
\bar{B} P=P B
$$


Как и блочные кватернионы, матрицы $B$ унитарно подобны своим (поэлементно) сопряженным матрицам $\bar{B}$.

Заметим, что матрица $P$, удовлетворяющая соотношениям (16), может быть посредством унитарной конгруэнции преобразована в матрицу $J$ (см., например, [2; задача 4.4.26]):

$$
U P U^{T}=J, \quad U U^{*}=I_{n} .
$$

Переписывая (18) в виде

$$
\left(U \bar{B} U^{*}\right)\left(U P U^{T}\right)=\left(U P U^{T}\right)\left(\bar{U} B U^{T}\right),
$$

заключаем, что унитарное подобие

$$
B \rightarrow A=\bar{U} B U^{T}
$$

переводит матрицы $B$ из класса (18) в блочные кватернионы. Отсюда и из проведенного выше обсуждения мы делаем следующий вывод.

ПРЕДЛОЖЕНИЕ 1. Если матрица В из класса (18) унитарно подобна вещественной матрице, то В приводима.

3. Доказательство теоремы 1. Необходимость условия теоремы почти очевидна. В самом деле, пусть матрица

$$
B=Q^{*} A Q
$$

вещественна, а матрица $Q$ унитарна. Тогда

$$
B=Q^{T} \bar{A} \bar{Q}
$$

и

$$
\bar{A}=\bar{Q} Q^{*} A Q Q^{T}=P^{*} A P,
$$

где

$$
P=Q Q^{T}
$$

Таким образом, матрица $P$, трансформирующая $A$ в $\bar{A}$, не только унитарна, но и симметрична.

Перейдем к доказательству достаточности. Пусть

$$
\bar{A}=P^{*} A P,
$$

где $P$ - унитарная симметричная матрица. Поскольку $A$ и $\bar{A}$ унитарно подобны, спектр матрицы $A$ должен удовлетворять условиям а) и б). Поэтому $A$ можно представить в виде

$$
A=Q M Q^{-1}
$$

где $M$ - вещественная матрица (например, вещественная жорданова форма матрицы $A)$. Подставляя (20) в (19), имеем

$$
\bar{Q} M \bar{Q}^{-1}=P^{*} Q M Q^{-1} P
$$


или

$$
\left(Q^{-1} P \bar{Q}\right) M=M\left(Q^{-1} P \bar{Q}\right)
$$

Положим

$$
D=Q^{-1} P \bar{Q} .
$$

Таким образом,

$$
D M=M D .
$$

Заметим, что

$$
D \bar{D}=\left(Q^{-1} P \bar{Q}\right)\left(\bar{Q}^{-1} \bar{P} Q\right)=Q^{-1} P \bar{P} Q=I_{n} .
$$

Последнее равенство объясняется тем, что

$$
P P^{*}=I_{n}, \quad P=P^{T}
$$

и, следовательно,

$$
P \bar{P}=I_{n} .
$$

Согласно теореме 4 матрица $D$ может быть представлена в виде

$$
D=S \bar{S}^{-1}
$$

Подставляя это выражение в (22), находим

$$
S \bar{S}^{-1} M=M S \bar{S}^{-1}
$$

или

$$
\bar{S}^{-1} M \bar{S}=S^{-1} M S .
$$

Таким образом, матрица

$$
L=S^{-1} M S
$$

вещественна.

Подставим выражение (24) и в (21). Имеем

$$
P=Q D \bar{Q}^{-1}=Q S \bar{S}^{-1} \bar{Q}^{-1}=V \bar{V}^{-1},
$$

где

$$
V=Q S
$$

Поскольку все сингулярные числа унитарной матрицы $P$ равны единице, ее факторизация Такаги имеет вид

$$
P=U U^{T}, \quad U U^{*}=I_{n}
$$

(см. теорему 5). Итак,

$$
V \bar{V}^{-1}=U U^{T}
$$

или

$$
U^{*} V=U^{T} \bar{V}
$$

т.e.

$$
R=U^{*} V
$$

есть вещественная матрица. 
Используя (20), (25) и (26), получаем

$$
A=Q M Q^{-1}=V S^{-1} M S V^{-1}=V L V^{-1}=U\left(R L R^{-1}\right) U^{*} .
$$

Матрица $R L R^{-1}$ вещественна, а $U$ унитарна. Таким образом, $A \in \mathscr{U} \mathscr{R}_{n}$ и теорема 1 доказана.

4. Доказательство теоремы 3. Пусть

$$
\bar{A}=P^{*} A P, \quad P P^{*}=I_{n} .
$$

Отсюда

$$
A=P^{T} \bar{A} \bar{P}=P^{T} P^{*} A P \bar{P}
$$

т.e.

$$
Q A=A Q
$$

где матрица $Q=P \bar{P}$ унитарна. Если $Q$ не является скалярной матрицей, то равенство (27) противоречит неприводимости матрицы $A$. Действительно, в этом случае $Q$ имеет нетривиальную пару взаимно дополнительных ортогональных подпространств, отвечающих различным частям ее спектра. Эта пара подпространств приводит и матрицу $A$.

Итак, $Q$ должна быть скалярной матрицей и, следовательно, имеет единственное собственное значение. Учтем теперь следующие особенности спектров матриц вида $Q=P \bar{P}$ (см., например, [2; задачи 4.6.6, 4.6.7]):

1) если $\lambda$ является собственным значением матрицы $Q$, то $\bar{\lambda}$ - также собственное значение;

2) если $Q$ имеет отрицательное собственное значение, то его кратность должна быть четной.

Свойство 1) означает, что в равенстве

$$
Q=\alpha I_{n}
$$

число $\alpha$ обязательно вещественно. Свойство 2) и условие теоремы 3 о нечетности порядка $n$ исключают случай $\alpha=-1$. Следовательно,

$$
Q=P \bar{P}=I_{n} .
$$

Соединяя это с равенством $P P^{*}=I_{n}$, мы заключаем, что матрица $P$ симметрична. Теперь нужное утверждение вытекает из основной теоремы 1. Теорема 3 доказана.

ЗАмЕЧАНИЕ. При четном $n$ равенство

$$
Q=-I_{n}
$$

возможно. В этом случае $A$ является либо блочным кватернионом, либо матрицей, унитарно подобной некоторому блочному кватерниону (см. п. 2). Если такая матрица принадлежит классу $\mathscr{U} \mathscr{R}_{n}$, то она с необходимостью приводима и, следовательно, включение $A \in \mathscr{U} \mathscr{R}_{n}$ никак не может быть выведено из теоремы 3.

Условие неприводимости исключает применение теоремы 3 к таким матричным классам, как нормальные матрицы или матрицы с квадратичными минимальными многочленами. Однако эти два матричных класса принадлежат $\mathscr{U} \mathscr{R}_{n}$, что из других соображений показано в [5]. 


\section{СПИСОК ЦИТИРОВАННОЙ ЛИТЕРАТУРЫ}

[1] Г. Е. Шилов, Математический анализ. Конечномерные линейные пространства, Наука, М., 1969.

[2] Р. Хорн, Ч. Джонсон, Матричный анализ, Мир, М., 1989.

[3] C. Pearcy, "A complete set of unitary invariants for operators generating finite $W^{*}$-algebras of type I", Pacific J. Math., 12 (1962), 1405-1416.

[4] Х.Д. Икрамов, "О конечном рациональном критерии неприводимости матриц", Вестн. Моск. ун-та. Сер. 15. Вычислит. матем., кибернет., 2007, № 3, 16-18.

[5] Х. Д. Икрамов, "О комплексных матрицах, овеществляемых посредством унитарного подобия", Докл. РАН, 430:1 (2010), 15-17.

Х. Д. Икрамов

Поступило

Московский государственный университет

им. М. В. Ломоносова

E-mail: ikramov@cs.msu.su
03.08.2009

Исправленный вариант

01.12.2009 